\title{
Habits, routines and temporalities of consumption: from individual behaviours to the reproduction of everyday practices.
}

Dale Southerton (The University of Manchester)

\section{Abstract}

The terms habit and routine have come to be used with increasing frequency in debates about 'behaviour' change and sustainable consumption, where dominant approaches, dubbed as 'portfolio models of action' by their critics, employ these terms to capture human deficiencies in the translation of pro-environmental values into corresponding actions (the 'value - action' gap). Alternative approaches present habits and routines as the observable performances of stable practices. Informed by these approaches, this article makes three 'conceptual moves' in order to demonstrate the need for empirical attention to the temporal conditioning of everyday practices. First, it is argued that conceptual usages of the terms habit and routine are often imprecise and used generically to capture many different aspects of human action. A three-fold conceptual framework comprising of 'dispositions', 'procedures' and 'sequences' is proposed as a preliminary step in dealing with this problem. Second, it is suggested that generic uses of the terms habit and routine imply multiple forms of temporality. Again, as a conceptual sorting exercise, three categories of temporality pertinent to understanding the performance of practices are examined: time as a resource; the temporal demands of practices; and, temporal rhythms. Third, the relationships between the conceptual variants of habitual and routine actions and temporalities of practices are examined through reference to empirical research. In conclusion, it is argued that reducing habits and routines to generic descriptions of behaviour within portfolio models of action is inadequate for developing understandings of the reproduction of everyday practices. Rather, empirical and 
conceptual attention to the relationships between practices, temporalities and different forms of action are required if the challenge of fostering more sustainable ways of life is to be met.

Keywords: habits; routines; time; sustainable consumption; behaviour; practices.

\section{Introduction}

Contemporary forms, and volumes, of consumption in advanced capitalist societies are widely identified as a principal source of human-induced climate change (Munasinghe et al., 2009). Understandably, policy and academic debates have thus come to focus on changing consumer behaviour as a fundamental remedy for this social problem. Such debates have been dominated by particular approaches to consumption and behaviour. These approaches are located within what Whitford (2002: 325) refers to as 'the portfolio model of the actor', a model in which 'individuals carry a relatively stable and pre-existing set of beliefs and desires from context to context. Given the situation, they select from this portfolio "those elements that seem relevant and [use] them to decide on a course of action".' Such a model applied to consumption presents the individual as a sovereign consumer, where consumption is largely a matter of personal decision-making within commercial markets that, in turn, respond to (aggregate) consumer demands and provide the sovereign consumer with a range of product choices from which to shape their lifestyle (Southerton et al., 2004). Within the portfolio model of action, consumer choices are the outcome of an individual's values and attitudes. The implications of such causal models are simple enough, to change behaviour (for example, towards more sustainable ways of life) requires changes in the attitudes that shape, and values that frame, consumer choices (Shove, 2010).

The problem, however, is that changing attitudes and values does not necessarily lead to a change in what people consume or, more importantly with respect to sustainability, the resource-intensity of 
their consumption. This is captured in the much used phrase 'value - action' gap in which people's reported positive attitudes towards the environment are not matched by their behaviours. This 'gap' is often explained as being the consequence of habits and routines, variables that complicate rational responses to policy initiatives (Hobson, 2003). More recently the notion of habits and routines as obstacles to individual actions has received renewed vigour through behavioural economics and the idea of 'nudge'. Drawing from cognitive science understandings of the brain as having two systems (one which is uncontrolled, unconscious and fast; the other reflective, controlled, deliberative and slow), Thaler and Sunstein (2008) suggest that much behaviour is governed by automatic and intuitive mental processes (the second system). That such behaviour is not rational results in bias actions and social conformity, which can be described as habitual (see, for example, Dolan et al., 2010). Such approaches thus advocate changing the default formats (the contextual cues) of behaviours (such as switching organ donation schemes from automatically being opted out to in) in order to overcome inertia and nudge habits in particular directions. Among the critiques of this approach is that the portfolio model of action, particularly theories of rational choice and planned behaviour, remain the standard against which all other forms of behaviour are judged; habitual action is reduced to little more than automated responses to contextual stimuli; and, habits are presented as ubiquitous, uniform and universal modes of action across social groups (see, for example, Hodgson (1997) on the ubiquity of habits). In short, habitual or routine actions (or behaviours) are reduced to deficiencies of the dominant portfolio model of human action.

Habit, however, is a word with many meanings. According to the Oxford English Dictionary (OED), it can be used to describe: 'a settled or regular tendency or practice'; practices that are 'difficult to give up'; automatic reactions to a given situation; specific forms of dress; a 'bodily constitution'; and, addiction, where repetitive behaviour becomes a psychological and/or physiological condition that produces an individual pathology. Habit is often conflated with the related word routine: 'a regular course or procedure'; 'a set sequence in a performance'; a duty; and a 'customary or standard' form 
of behaviour (OED). As such, the terms habit and routine capture the performance of everyday forms of action that appear to exhibit, in various combinations, shared cultural conventions, recurrent, and non-reflexive behaviour.

This article seeks to develop a preliminary conceptual framework for analysing variant forms of action that are often described as habitual and routine behaviour. It presents a preliminary framework because, as with all conceptual developments, empirical interrogation is necessary to test and develop the theoretical propositions that underpin it. Informed by theories of practice as applied to the study of consumption, the article begins with the claim that habitual and routine forms of action are the observable performances of stable practices. However, the wider use of the terms habit and routine obscure analysis of how practices are reproduced. Habits and routines have become generic terms that variously capture apparently non-reflexive (or automatic), repetitive and recurrent actions. As a first 'conceptual move', this article presents a conceptual framework that identifies three variants of action that are often conflated by the descriptive use of the terms habits and routines: dispositions, procedures, and sequences. A second conceptual move reviews the recursive relationship between temporalities and the performance of practices: temporalities configure the performance of practices and, in turn, the performance of practices shape temporalities. The final section, and third conceptual move, seeks to reconcile the temporalities of practices with three variants of habitual and routine actions by considering empirical accounts that shed light on how dispositions, procedures and sequences of action configure the performance of practices. In conclusion it is argued that changing practices in directions that foster more sustainable forms of consumption requires a radical reconceptualization of human action (away from portfolio models and towards practice-based approaches), and greater empirical attention to temporalities and how they interact with dispositions toward, and procedures and sequences of, the performance of practices. 


\section{Consumption and Practices: habits and routines}

In his reflections on the sociological contours of the field, Warde (2010) presents a schematic outline of three fundamental dimensions of consumption, each of which broadly correspond with different phases of intellectual scrutiny: acquisition; appreciation; and, appropriation. The first was attention to the acquisition of goods and services focused around debates concerning welfare provision, the distribution of resources, and the constitution of needs and wants. The 'cultural turn' in the social sciences of the 1970s and 1980s turned analytic attention toward appreciation, with emphasis on the symbolic properties of goods, the pleasures of consumption, and its capacities for selfexpression and communication. The third dimension, which has gained prominence since the 1990s, draws attention toward processes of appropriation and material culture, with a focus on how goods and services are incorporated, adapted and used for practical purposes. Among the notable features of this third phase is a shift in analytical attention away from conspicuous and highly symbolic forms of consumption toward accounts of inconspicuous, ordinary and mundane forms of everyday consumption (Gronow and Warde, 2000). It is through growing attention to the mundane use of things, particularly in relation to debates about sustainable consumption, that sociological interest in habitual and routine forms of action and theories of practice have emerged.

Theories of practice are, by common consent, very diverse (Schatzki, 2011). Emerging in the 1970s, they are identified with a heterogeneous set of authors, prominent among them (according to Ortner, 1984; Schatzki 1996) being Bourdieu, Giddens, Sahlins, Foucault, Lyotard, Garfinkel, Charles Taylor and Judith Butler. The dominant view is that this development was a diverse response to fundamental and pressing problems of social theory like structure and agency, the role of rules in social order, and the scientific status of social theories (Ortner, 1984; Rouse, 2006). Renewed attention was signalled by the rhetorical announcement in 2001 of 'the practice turn' in 
contemporary social theory (Schatzki et al., 2001). One key feature of the reformulation in the later 1990s was to proclaim practices the fundamental unit of social analysis and to suggest that the recursive relationship between individual performances and social practices could provide a sound basis for empirical social science. Schatzki (1996) was the most systematic exponent, but Reckwitz (2002) was also particularly important for the advancement of a sociological approach. Since the turn of the century, the concept of practice (and various derivatives) has circulated widely, and been applied variously in empirical studies which have described, interpreted and explained social processes and behaviour in specific domains (Postill, 2010). The domain of consumption is no exception, and the Journal of Consumer Research and the Journal of Consumer Culture (see especially, Warde 2005, and Halkier, Katz-Gerro and Martens, 2011) both have carried several articles since 2005.

The idea that practices are social and shared is commonly agreed, though explanations of the degrees to which practices are shared is contentious, depending on whether practices are considered as entities in themselves or are merely similar performances repeated widely across time. When operationalised, practices are generally treated as configurations of recognizable, intelligible and describable elements which comprise their conditions of existence. While there is no single agreed typology of elements (compare options in Gram-Hansen, 2011, and Shove et al., 2012), some combination of material objects, practical know how, and socially sanctioned objectives is deployed, these often in context of socio-technical systems, social and economic institutions, and modes of spatial and temporal organization. Such elements and contexts both configure how practices are performed and make them identifiable to practitioners and non-practitioners alike. The relationship between practices and performances is recursive: practices configure performances, and practices are reproduced and stabilized, adapted and innovated, through performances. A critical theoretical question thus focuses on the tensions and dynamics between the reproduction (stability) of stable practices and adaptation (innovation) in the performance of practices that hinder 
or generate social change. Practices as stable entities reproduced through faithful performances are often described as habitual and routine practices (e.g. Shove, 2012), and it is these faithful performances of practices that need to change if patterns of consumption are to shift in more sustainable directions (Evans et al., 2012).

Such an approach to habits and routines is largely consistent with other applications of the terms in the social sciences. From his review of the literature, Swartz (2002), for example, outlined the key characteristics of habits as: predictability and regularity of particular actions; a unifying force of action within and across socio-cultural groups; collectively derived actions; a conservative force; and, conditioned by institutions. Camic (1986: 1044), in his classic essay on habit, settled on the following definition: 'a more or less self-actuating disposition or tendency to engage in a previously adopted or acquired form of action'. Added to this is the term routine, which is often conflated with that of habit due to its similar emphasis on non-reflexive action. Perhaps the core difference can be found in the implication of routines as activities that follow a particular or familiar sequence, recur with relatively predictable periodicity or, as is the case in the concept of organizational routines, which represent institutionalised and tacit procedures that guide actions and produce 'recurrent interaction patterns' (Becker, 2005).

Conceptual use of the terms habit and routine is obscured by both seeking to capture three related observations, that: many everyday actions are performed with a high degree of recurrence, periodicity and a degree of predictability; much action appears to be performed without reflexive deliberation; and, many such actions are culturally shared whether across whole societies or social groups. The first two observations are most closely associated with approaches that present habits and routines as remnants of past reflexive deliberations that re-occur as necessary mental short-cuts for navigating the complexities of daily life - Giddens' (1984) concept of practical consciousness being a prominent example. Such perspectives, despite their sociological application, have their 
roots in psychological stimulus-response models of action, where habits and routines are presented as behaviours that occur with a high degree of frequency, are automatic and occur repeatedly in stable contexts (Neal et al., 2006). Automaticity is triggered by external environmental stimuli, meaning that habits and routines are defined as the: 'learned sequences of acts that have become automatic responses to specific cues, and are functional in obtaining goals or end states' (Verplanken et al., 2005). The emphasis is that such acts were, at one point, conscious and reflexive but lapse into automaticity. As such, a critical foci of empirical study is to identify opportunities to change the 'specific cues' that trigger habitual and routine actions so that they are raised back to a level of reflexive consciousness, thus allowing those habits to be re-set; what Verplanken et al. (2008) call 'habit discontinuity'. Non-reflexive and recurrent actions are therefore presented as a form of necessary 'auto-pilot' where the goals and values of any action do not need re-assessment unless the contexts of action change.

Accounts of action within cultural sociology take a rather different view, and tend to place greater emphasis on the second two observations (non-reflexive and culturally shared actions). Swidler's critique of the value-led model of action suggests that, 'culture influences action not by providing the ultimate values toward which action is oriented, but by shaping a repertoire or "tool-kit" of habits, skills, and styles' (Swidler, 1986: 273). While not discussing habitual action directly, this approach argues that culture provides procedures for actions without the requirement of individuals to reflexively deliberate about those actions. Such an approach leads Lizardo (2012) to present a 'procedural theory of acculturated action'; where procedures for action are assimilated from past experiences of practices. Changing such forms of action is, from such a perspective, less a matter of changing external environmental stimuli (as suggested by the habit discontinuity hypothesis), and more a matter of understanding how procedures are learned and acquired through recurrent performances of practices. 
Given these conceptual vagaries it appears that limited conceptual mileage can be gained from attempting to distinguish between the terms habit and routine or to attempt a singular definition. Rather, it is more instructive to recognise that habits and routines refer to conceptually distinct, if inter-related, forms of everyday action. Warde and Southerton (2012: 20) suggest a conceptual framework that presents three forms of action that are generically captured by the terms habits and routines. The first, most closely associated with the term habitus (Bourdieu, 1984), refers to dispositions: 'a propensity or tendency to act in a particular manner when suitable circumstances arise. Dispositions can provide an impetus to action both in situations which do not necessarily occur very frequently and, by virtue of transposition, in situations not previously encountered'. Dispositions can be thought of as culturally derived and shared orientations towards the performance of practices. The second refers to procedures: 'previously learned and ready to hand, waiting to be drawn upon when appropriate circumstances present themselves'. Procedures, often observed as non-reflexive actions, capture the taken for granted forms of tacit knowledge and embodied skills that relate to the performance of any given practice. The final third conceptual form refers to sequences of activity: 'guided by social signals or by equipment which more or less orchestrate' the performance of practices. Sequences produce recurrent actions through institutional and material scripting of practices.

Understanding habits and routines as generic terms that variously capture dispositions toward, and procedures and sequences of, practice performances, presents a useful conceptual starting point for explaining apparently non-reflexive and recurrent actions without recourse to an ontological position which posits individualised defects to the portfolio model. Presented this way, habitual and routine actions are understood as the observable performances and patterning of stable practices, and the critical question becomes how such practice performances are held stable. Adam's (1998) 'timescapes' conceptual framework presents temporalities as one set of mechanisms that order everyday practices and experiences. That many accounts of habits and routines imply the 
significance of temporalities with respect to periodicity and sequences further suggest them to be an important mechanism. This raises an important set of questions to which this article now turns: what is the relationship between practices and temporalities; to what extent might temporalities hold practices stable; and, to what extent might the temporalities of practices relate to the different conceptual forms of habitual and routine action.

\section{Practices and Temporalities}

Whether temporalities configure practices or practices effectively shape temporalities and temporal experiences is a matter of debate. Shove et al. (2012) usefully observe that experiences of time are experiences of practices; the passing of time, tempos, repetition, recollection of past times and anticipation or imaginations of the future are mediated by the practices through which that time is experienced. Beyond this broad observation a parsimonious categorization of the relationship between temporalities and practices can be presented through three themes: time as a resource; practices as configuring temporalities; and temporal rhythms.

That time is a resource, much like money, used or spent in relation to different practices is an uncontroversial observation. Indeed, the field of time-use studies represents a significant research endeavour devoted to understanding the changing, and often unequal, distribution of practices in and across time. Treating time as a finite, objective, resource and analysing it in zero-sum terms has produced some critical understandings of consumption and social change. Schor's (1992) argument that contemporary Americans work more to consume more is among the most influential studies that explores the allocation of time as a resource across practices. Using a range of economic and time diary data she shows that the US economy has, year on year, increased its productivity rate meaning that more goods and services are produced in the same amount of time. By extension, the possibility emerges of working less time for the same productive output. This is not what has 
happened. Instead, the average American is working longer hours and consuming increasingly more. This, Schor explains, is a consequence of the desire for more consumption (which requires more work) fuelled by media saturation of affluent lifestyles to which people aspire and use as a relative measure of their own well-being. Similar studies question the extent to which one might measure and substitute between different categories of practices such as work and leisure consumption (e.g. Robinson and Godbey, 1996), and demonstrate more nuanced trends and patterns in the allocation of practices within time for other countries (e.g. Gershuny, 2000). However, the fundamental starting point for such accounts is that practices compete for time within the 24 hour day, seven day week and 365 day year.

In her discussion of habitual practices, Shove (2012) argues that practices (as entities) need to colonize time slots in order to taken on habitual and routine forms. In this respect, not only do practices compete for time but also for temporal locations with the day, week, month, and so on. Such an analysis suggests that to unsettle or change practices that have habitual or routine characteristics requires disrupting the allocation of time across those practices. Such an approach is novel with respect to understanding the formation and reproduction of habitual actions, but the idea of re-distributing practices in time is not. Gershuny's (2000) account of 'humane modernization' and Schor's (2010) account of wealth and consumption both identify changes to the working week (the reduction of hours of work) as mechanisms for unlocking and re-calibrating the relationship between time as a resource and everyday practices in ways that could lead to a more progressive development of the economy, well-being and, in Schor's case, environmental sustainability.

A second theme around which the relationship between practices and temporalities can be categorized is through a consideration of how practices configure temporalities. In my empirical analysis of the temporal ordering of practices (Southerton, 2006), I demonstrated how different practices produce their own temporal demands based upon the degree to which they require 
coordination (or synchronisation) with other people or practices. Such temporal demands are best explained by considering the inter-related temporal dimensions of duration, tempo, sequence and periodicity. Some practices, such as eating meals with friends and family, required a high degree of coordination with the schedules of co-participants and, as a result, were often located within specific sequences of related practices (such as food provisioning and preparation). In these cases, practices required (or demanded) particular temporal conditions for their performance. By contrast, practices less dependent on particular forms of purposeful coordination, for example watching television and reading, presented fewer temporal demands. Such practices, partly as a consequence of high degrees of coordination, were located in particular time frames (typically evenings and weekends), and were performed with a particular tempo and duration. The differing temporal requirements for the satisfactory performance of practices have the effect of ordering the temporal rhythms of everyday life.

Returning to Shove's suggestion that practices compete for time, this second set of relationships emphasise that practices come with their own temporal demands which condition both the experience and the performance of those practices. Practices are not simply distributed in time (and space). Rather, the temporal characteristics of some practices lend themselves to particular locations within time more so than do others (such as dinner parties on weekend evenings as opposed to weekday lunchtimes). It follows that the formation and reproduction of 'particular' practices that appear to take on habitual and routine forms of action are, at least in part, likely to be conditioned by the multiple temporal demands of those practices.

The third category for conceptualising the relationship between practices and temporalities refers to collective and personal temporal rhythms. This category represents a reversal of the second: temporal rhythms configure practices. Societies, to differing extents, have collective temporal rhythms that are easily identified. Peak loads in energy consumption and transport use represent 
simple empirical observations that reveal such rhythms. Many collective temporal rhythms that condition the way practices are understood and performed have been identified in empirical studies, including: fashion cycles (Gronow, 2009); seasons (Daniels, 2009); bodies (Adam, 1995); television programming (Silverstone, 1993); school and travel times (Skinner, 2005); meal times (Southerton et al, 2012); and, working times (Brannen, 1995). The extent to which collective temporal rhythms condition practices across different societies or whether such conditioning has effectively been eroded remains open to debate. Empirical analysis of the contrasting temporal rhythms of UK daily life in 1937 and 2000 reveals that collective temporal rhythms (symbolized by Sunday dinners and Monday's as wash day) appear to have been weakened by greater flexibility of institutionally timed events (related to working, shopping and eating times) and the changing technological infrastructures of UK homes (Southerton, 2009). Other studies suggest that collective temporal rhythms have been eroded by the emergence of the $24-7$ society and the temporal acceleration of everyday life (Rosa, 2003). Collective temporal rhythms, under such circumstances, are superseded by personalized temporal rhythms that involve time-deepening strategies of doing practices faster, simultaneously, substituting practices of short for long durations, and more detailed inter-personal time planning (Robinson and Godbey 1996).

Regardless of whether collective temporal rhythms have been undermined and superseded by personal time strategies, this category highlights that temporalities are not only the outcome of the competition between practices for time or the temporal demands made by practices. Temporal rhythms frame practices and condition how they are performed. The relationship between practices and temporalities should therefore be understood as recursive and mutually inter-dependent. It follows that if habitual and routine forms of action are the observable performances of stable practices, then the three categories of relationship between practices and temporalities are likely to play an important role in framing and holding in place those actions. It is to this proposition that this 
article turns next in order to examine how the temporalities of practices relate to the dispositions, procedures and sequences of action.

Temporalities and practices: dispositions, procedures and sequences

The argument so far has been that habitual and routine forms of actions are observable in the performances of stable practices. Temporalities are a critical mechanism that hold practices stables, although the relationship between practices and temporalities should be understood as recursive: temporalities shape practices and practices shape temporalities. (For ease of discussion, this recursive relationship will be referred to as the temporalities of practices from here on.) It has also been argued that habitual and routine forms of action are generic terms that encompass three conceptual variants. It follows that to understand forms of action generically described as habitual and routine it is necessary to explore in more detail the relationships between the temporalities of practices and dispositions toward, and procedures and sequences of, action.

\section{Temporalities and dispositions}

The concept of dispositions is most readily associated with the work of Bourdieu (1990) and his associated use of the concept habitus. The habitus is a system of acquired dispositions, which represent durable and transposable courses of action that are not deliberative or formed through express intentions or calculations yet remain purposive. Critically, for Bourdieu, the habitus maps onto objective group positions within social space, although its associated dispositions are constantly modified by experience and trajectory in ways that offer forms of coherence (homology) of actions across practices . In his account of social distinction, Bourdieu (1984) argues that dispositions, as reflected through tastes in consumption, emanate from the pursuit of the conduct of a life which is subjectively acceptable in the context of objectively given circumstances related to material wealth (economic capital), types of knowledge (cultural capital), and forms of social 
networks (social capital). In this respect, dispositions explain Bourdieu's empirical observation that people in similar objective social positions have similar dispositions toward sets of practices that transpire into recognisable, recurrent and socially shared patterns of action.

Holt's (1997) analysis of dispositions towards practices associated with clothing, home décor, travel, watching movies, reading, eating and socialising in the USA is particularly useful for identifying sets of transposable dispositions. He identified four dispositional antinomies that differentiated the people that he interviewed: autotelic versus self-actualising pursuit of practices; favouring function and utility over style; conformity versus originality and individuation; and, critical versus referential appreciation. Those with high volumes of economic and cultural capital had a tendency to deploy dispositions that were self-actualising, stylized, which embraced originality and individuation, and employed forms of critical appreciation when describing their orientations toward the performance of practices. Those with lower volumes of capital had tendencies toward the opposite poles of the dispositional antinomies. For Holt, the critical qualification is that dispositions are not simply about what people do, in his study those with high and low volumes of capital engaged in the same sets of practices and, in some cases, consumed the same goods and services (e.g. watched the same movies), but did so in different ways, with varying meanings and distinct purposes. What might appear as habitual actions through the patterning of practices mask very different dispositions towards the performance of those practices.

There are two ways of conceptualising the relationship between the temporalities of practices and dispositions. The first is to consider how dispositions affect the allocation of practices in time. As the earlier discussion of time as a resource implied, how different social groups allocate practices within time is revealing about patterns of consumption. That the professional middle classes appear to hold dispositions that result in the devotion of more time to practices such as eating out, reading books and relatively less time to watching television than other social groups (Gronow and Southerton, 
2009), presents a basic observation that dispositions affect the patterning of practices (and, therefore, consumption) in time. More insightful observations can, however, be found in studies that explore dispositions towards the use of time more generally. For example, Gershuny's (2005) account of 'busyness as a badge of honour' suggests that certain social groups hold dispositions that encourage a high tempo and intensity of the performance of practices; studies of experiences of harriedness identify dispositions related to the fear of wasting time and which favour rigid allocations of practices to particular time slots in the day as shaping the temporal rhythms of everyday practices (Southerton, 2003); and, studies of workplaces reveal differential dispositions towards the maintenance of clear boundaries between work and domestic practices as having significant implications for the temporal experience of those practices (Brannen, 2005).

The second approach is to consider how the temporal demands of practices are shaped by dispositions toward their satisfactory performance. Dispositions that favour self-actualization, as opposed to the autotelic performance of any given practice, are likely to result in practice-based demands for the devotion of greater durations, frequency (or periodicity) and, perhaps, for more detailed coordination with co-participants. This is, for example, observed in empirical studies of practice enthusiasts, such as gastronomes who stand out in empirical accounts of the practice of eating because they exhibit longer durations, slower tempos, and greater frequencies of eating out than do other social groups (Warde et al., 2007). While observations of patterned and repetitive performance of practices are often described as being habitual and routine, few studies pay direct attention to how differential dispositions towards the performance of those practices relate to the temporalities of practices. The result is mere description of actions through the observation of recurrence and durations, as opposed to any systematic analysis of why those temporal patterns come to take on their particular form and what role they might play in reproducing practices. 


\section{Temporal procedures}

Procedures refer to the forms of tacit knowledge and embodied actions that underpin the performance of any practice in ways that neither require nor lend themselves to cognition. According to Zerubavel (1981) temporal regularities provide a blueprint (or temporal map) for procedural performances of practices. His account begins with the observation that contemporary societies are remarkable for the degree to which they are ordered and regulated by time. He outlines four principles of temporal regularity that, in the language employed in this article, can be re-interpreted as temporal dimensions that frame taken-for-granted procedures for the performance of practices.

Zerubavel's first principle is 'rigid sequential structures'. He observes that most events in daily life cannot take place simultaneously and must be temporally segregated as being 'before' or 'after', and many sequential structures are rigid and irreversible. Some of this rigidity is a consequence of natural or technical procedure (see 'temporal sequences' below), others relate to culturally derived conventions regarding the acceptable sequence through which practices might be performed, such as the temporal stages of intimate relationships. Second are tacit understandings and knowledge of the 'expected duration' of activities. Zerubavel provides examples of people feeling cheated or restless when the durations expected do not fit the conventions of the practice, such as concerts and movies that do not last around two hours or people under- or over-staying their welcome. Zerubavel's third principle refers to practices as having 'standardized temporal locations'. Particular foods (and drinks) are typically taken at certain moments of the day, and there are appropriate contexts for being early or late for an arrangement. Finally, many practices have a 'uniform rate of recurrence', as illustrated by patterns of eating and personal hygiene (e.g. brushing one's teeth).

For Zerubavel (1981: 12) these four principles of temporal regularity produce 'a reliable repertoire of what is expected, likely, or unlikely to take place within certain temporal boundaries... [and]... adds 
a strong touch of predictability to the world around us'. Re-interpreted in light of theories of practice, such temporal regularities coordinate and hold practices stable in ways that generate procedures of action. Such an interpretation resembles Zerubavel's (1981: 14) metaphor of temporal maps:

'One of the implications of the highly regular temporal profile of so many social events is that we carry in our minds a sort of "temporal map" which consists of all our expectations regarding the sequential order, duration, temporal location, and rate of recurrence of events in our everyday life.'

The metaphor of temporal maps is problematic as it implies fixed contours between temporal dimensions, but the argument that temporalities condition actions by providing tacit practical 'guidelines' for the performance of practices is more convincing. Consistent with accounts of habits and routines as practical consciousness, Zerubavel's analysis supports an argument that to shift procedural forms of action requires attention to the temporalities (or temporal principles) that hold in place stable practices.

\section{Temporal sequences}

Zerubavel's account of temporal sequence largely focuses on culturally derived conventions. Of equal significance are the material, infrastructural and institutional sequencing of practices. As the earlier discussion of temporal rhythms indicated, many sequences of activities are held in place by multiple technologies, infrastructures and institutionally timed events. Empirical debate about the extent and implications of the weakening of such temporal rhythms as mechanisms of collective coordination are, essentially, debates regarding the contemporary rigidity of the temporal sequencing of practice. While it may be the case that contemporary societies present less binding and more flexible sequences of practices, the continued existence of collective patterns of action suggests that temporal sequences remain salient. Sequences of performance can be identified as recurrent and non-reflexive actions, for example, in the technological affordances of material 
objects, such as oversized hotel key fobs or road traffic calming technologies (Latour, 1988) that set sequences which script actions - in these examples returning key fobs when leaving hotels and the slowing of vehicles at certain locations in road networks. Temporal sequences of action are also embedded in infrastructures of various forms, such as the sequences of action scripted by laundry infrastructures (Shove, 2003), and institutionally timed events that frame sequences of practices such as patterns of food shopping on the way home from work and school drop offs on the way to work (Skinner, 2005).

\section{Conclusion}

This article began by noting the growing public, policy and academic focus on consumption as a fundamental domain of daily life where change is needed in order to tackle human-induced climate change. It was argued that dominant models of action frame consumption largely as a matter of individual discretion, and casts the subject of debate in terms of informing and encouraging consumers to make more sustainable lifestyle choices. Habits and routines have risen to the top of the agenda in response to the value-action gap, which appears to confound those dominant models of action. Yet, habits and routines have come to be presented as human deficiencies of the portfolio model of action rather than as a challenge to theoretical orthodoxy. This article has sought to present an alternative model of action, to present some conceptual clarification of the generic terms habit and routine, and has argued that the temporalities of practice both configure and are conditioned by dispositions toward, and procedures and sequences of, action.

Theoretical approaches to understanding human action as being embedded in practices place emphasis on analysing how practices are configured to form recognisable entities that are performed in everyday life. This is a recursive relationship. Practices structure performances (by constraining the range of possible alternatives) and through their performance practices are 
reproduced in particular forms. Habits and routines reflect stable practices as entities that permit and are reproduced through recurrent, non-reflexive, and culturally shared actions. Explained this way, habits and routines are the observable patterns of action that result from the reproductive performances of stable practices. It follows that to analyse and explain these observable actions a more robust conceptual framework that captures the different forms of action generically described as habits and routines is necessary. As a first step, this article has presented a three-fold conceptual framework, consisting of: dispositions - the tendency to perform practices according to culturally derived orientations; procedures - tacit knowledge and embodied skills; and, sequences - the ordered performance of practices through material, infrastructural and institutional forms.

A fundamental feature of both the configuration and performance of practices, especially those that can be observed as taking habitual and routine forms, are temporalities. The recursive relationship between practices and temporalities is captured by considering how practices compete for time as a resource, how practices configure temporalities, and how collective and personal temporal rhythms frame and configure practices. Presented as a parsimonious categorization of the recursive relationship between temporalities and practices, these conceptual sketchings suggest that the temporalities of practices represent an instructive analytical theme for developing understandings of everyday life, consumption and particular forms of human action.

This suggestion was tentatively examined by exploring how the temporalities of practices interact with dispositions towards, and procedures and sequences of, actions in the performance of practices. Of particular importance in such an exploration was to consider how temporalities contribute to the reproductive performances of practices necessary to stabilise those practices. Dispositions were presented as mechanisms that affected the temporal allocation and distribution of practices across social groups, and which help explain variations between social groups in terms of the durations, tempos, periodicity and degrees of coordination attributed to the performance of 
practices. Such an analysis moves beyond the simple empirical observation that many actions are recurrent and patterned across social groups to argue that the variable temporalities of practices performed (and reproduced) by different social groups are shaped by culturally derived dispositions. The second conceptual form related to the procedures that underpin the performances of practices. Drawing upon Zerubavel's account of temporal regularities it was argued that cultural conventions of sequential order, duration, temporal location and rates of recurrence represent critical mechanisms which frame procedures of action. Finally, the sequential ordering of practices, especially through material, infrastructural and institutional settings, configure performances in ways often described as non-reflexive and recurrent actions.

The three-fold conceptual framework for disaggregated forms of action generically described as habits and routines clearly relate to the three thematic categories used to outline the relationship between practices and temporalities. The category of time as a resource connects with dispositions in terms of the allocation and distribution of practices in time. That practices create temporal demands has conceptual cross-overs with tacit understandings and embodied skills associated with procedures for practical performances. The sequential ordering of actions and thematic category of collective and personal temporal rhythms both emphasised how materialities, institutions and infrastructures configure the temporalities of practices and their sequential performance. However, it would be a mistake to present the three-fold conceptual framework and three thematic categories only in such a linear fashion. It is a matter for empirical research to explore and analyse the multiple relationships between the temporalities of practices and the different conceptual forms of action generically described as habitual and routine.

That such empirical endeavour is necessary should be beyond dispute. Recourse to deficiency accounts of the portfolio model of action reduce habits and routines to individualised obstacles for shifting consumption in more sustainable directions. Habitual and routine forms of action are more 
than a matter of individual deficiencies that need remedying. As practice-based approaches to consumption reveal, habits and routines are observable patterns of action that are socially conditioned and a fundamental basis of much (if not most) everyday action. The challenge, especially when applied to sustainable consumption, is to reconfigure such forms of action in ways that reduce the resource-intensity of everyday life. Attention to the temporalities of practices and how they relate to the conceptual forms of habitual and routine action presents one, instructive, avenue for exploring the scope and possibilities for such reconfiguration.

\section{References}

Adam, B. (1995), Timewatch: the social analysis of time, London: Polity.

Adam, B. (1998), Timescapes of Modernity: The Environment and Invisible Hazards, London: Routledge.

Becker, M. (2005), 'The concept of routines: some clarifications', Cambridge Journal of Economics, 29: $249-262$.

Brannen, J. (2005), 'Time and the negotiation of work-family boundaries: autonomy or illusion', Time \& Society, 14(1): 113-31.

Bourdieu, P. (1984), Distinction: A Social Critique of the Judgment of Taste, London: Routledge. Bourdieu, P. (1990), The Logic of Practice, Cambridge: Polity.

Camic, C. (1976,) 'The matter of Habit', American Journal of Sociology, 91(5) 1039-87.

Daniels, I. (2009), 'Seasonal and Commercial Rhythms of Domestic Consumption: A Japanese Case Study', in E. Shove, F. Trentmann \& R. Wilk (eds.) Time, Consumption and Everyday Life: Practice, Materiality and Culture, Berg: Oxford, pp 171-188.

Dolan, P., Hallsworth, M., Halpern, D., Kin, D. and Vlaev, I. (2010), Mindspace. Influencing Behaviour through Public Policy, London: The Institute for Government and Cabinet Office. 
Evans, D., McMeekin, A, \& Southerton D., (2012), 'Sustainable Consumption, Behaviour Change Policies, and Theories of Practice', in A. Warde \& D, Southerton (eds.), The Habits of Consumption, Helsinki: Open Access Book Series of the Helsinki Collegium of Advanced Studies. Gershuny, J. (2000), Changing Times: work and leisure in post-industrial society, Oxford: Oxford University Press.

Gershuny, J. (2005), 'Busyness as the badge of honor for the new superordinate working class', Social Research, 72(2): 287-314.

Giddens, A. (1984), The Constitution of Society, Cambridge: Polity.

Gram-Hanssen K (2011) 'Understanding change and continutity in residential energy consumption', Journal of Consumer Culture, 11(1), 61-78.

Gronow, J. (2009), 'Fads, Fashions and 'Real' Innovations: Novelties and Social Change', in E. Shove, F. Trentmann \& R. Wilk (eds.) Time, Consumption and Everyday Life: Practice, Materiality and Culture, Berg: Oxford, pp 129-142.

Gronow, J. \& Southerton, D. (2009), 'Consumption and Leisure in Europe' in G. Therborn \& S. Immerfell (eds.) Handbook of European Societies: Social Transformations in the 21st Century: A Reference to EU Societies and their Transformations in the 21st Century, Springer: London. Gronow, J. \& Warde, A. (eds) (2001), Ordinary Consumption, London: Routledge.

Halkier B, Katz-Gerro T and Martens L (2011) 'Applying practice theory to the study of consumption: theoretical and methodological considerations', Journal of Consumer Culture, 11(1), 3-14.

Hobson, K. (2003), 'Thinking habits into action: the role of knowledge and process in questioning household consumption practices', Local Environment, 8(1): 95-112.

Hodgson, G.M. (1997), 'The ubiquity of habits and rules', Cambridge Journal of Economics, 21: 663684.

Holt, D. (1997), 'Distinction in America? Recovering Bourdieu's theory of taste from its critics'. Poetics 25: 93-120. 
Latour, B. (1988), 'Mixing humans and non-humans together: The sociology of a door-closer'. Social Problems, 35, 298-310.

Lizardo, O. (2012), 'Embodied culture as procedure: rethinking the link between personal and objective culture', in A. Warde \& D, Southerton (eds.), The Habits of Consumption, Helsinki: Open Access Book Series of the Helsinki Collegium of Advanced Studies.

Munasinghe, M., Dasgupta, P., Southerton, D., McMeekin, A., Bows, A (2009), Consumers, business and climate change: Copenhagen 2009, Sustainable Consumption Institute.

Neal, D., Wood, W. and Quinn, J. (2006), 'Habits - a repeated performance', Current Directions in Psychological Science, 15(4), 198-202.

Ortner S (1984) 'Theory in anthropology since the Sixties', Comparative Studies in Society and History, 26: 126-66.

Postill J (2010)'Theorising media and practice', in Bräuchler B and Postill J (eds.) (2010) Theorising Media and Practice, New York: Berghahn Books, 1-34.

Reckwitz, A. (2002), 'Toward a theory of social practices: a development in culturalist theorizing', European Journal of Social Theory, 5(2): 243-63.

Robinson, J. and Godbey, G. (1997), 'Time for Life: The Surprising Ways that Americans Use their Time'. Pennsylvania: Pennsylvania State Press.

Rosa, H. (2003), Social acceleration: ethical and political consequences of a desynchronized highspeed society', Constellations, 10(1): 3-33.

Rouse, J (2006) 'Practice theory', in Handbook of the Philosophy of Science, vol.15: Philosophy of Anthropology and Sociology, (eds. S.Turner and M Risjrod), Elsevier, 500-540.

Schatzki, T. (1996)_Social Practices: a Wittgensteinian approach to human activity and the social, Cambridge: Cambridge University Press.

Schatzki, T., Knorr Cetina, K. and von Savigny, E. (eds.) (2001) The Practice Turn in Contemporary Theory, London: Routledge. 
Schatzki, T. (2011), 'Theories of Practice', in D. Southerton (ed.), Encyclopedia of Consumer Culture, Vol III, Thousand Oaks, CA: Sage, 1447-1551.

Schor, J. (1992), The Overworked American: The Unexpected Decline of Leisure. Basic Books.

Schor, J. (2010), Plenitude: The New Economics of True Wealth, New York: The Penguin Press.

Shove, E. (2003), Comfort, Cleanliness and Convenience: the social organization of normality, Oxford: Berg.

Shove E (2010), 'Beyond ABC: climate change policy and theories of social change, Environment and Planning A, 42: 1273-85.

Shove, E. (2012), 'Habits and their creatures', in A. Warde \& D, Southerton (eds.), The Habits of Consumption, Helsinki: Open Access Book Series of the Helsinki Collegium of Advanced Studies.

Shove, E., Pantzar, M. \& Watson, M. (2012), The Dynamics of Social Practice, London: Sage.

Silverstone, R. (1993), Time, information and communication technologies and the household, Time \& Society, 2(3): 283-311.

Skinner, C. (2005), 'Coordination points: A Hidden Factor in Reconciling Work and Family Life', Journal of Social Policy, 34(1): 99-119.

Southerton, D. (2003), “Squeezing Time': allocating practices, co-ordinating networks and scheduling society', Time \& Society, 12(1): 5-25.

Southerton, D. (2006), 'Analysing the temporal organisation of daily life: social constraints, practices and their allocation', Sociology, 40(3): 435-54.

Southerton, D. (2009), 'Temporal rhythms: comparing daily lives of 1937 with those of 2000 in the UK', in E. Shove, F. Trentmann \& R. Wilk (eds.) Time, Consumption and Everyday Life: Practice, Materiality and Culture, Berg: Oxford, pp 49-63.

Southerton, D., Warde, A. \& Hand, M. (2004), 'The Limited Autonomy of the Consumer: Implications for Sustainable Consumption', in D. Southerton, H. Chappells, \& B. Van Vliet (eds.), 'Sustainable consumption: the implications of changing infrastructures of provision', London: Edward Elgar, pp $32-48$ 
Southerton, D., Diaz Mendez, C., \& Warde, A. (2012), 'Behavioural Change and the Temporal Ordering of Eating Practices: A UK-Spain Comparison', International Journal of Sociology of Agriculture and Food, 19(1): 19-36.

Swartz, D.L. (2002), 'The sociology of habit: the Perspective of Pierre Bourdieu', The Occupational Therapy Journal of Research, 22: 61-69.

Swidler, A. (1986), 'Culture in action: symbols and strategies', American Journal of Sociology, 51: 273-86

Thaler, R. \& Sunstein, C. (2008) Nudge: Improving Decisions About Health, Wealth, and Happiness, New Haven, CT: Yale University Press.

Verplanken, B., Myrbakk, V. and Rudi, E. (2005), 'The measurement of habit', in T. Betsch and S Haberstroh (eds.), The Routines of Decision Making, Mahwah NJ: Lawrence Erlbaum, 231-247.

Verplanken, B., Walker, I., Davis, A. and Jurasek, M. (2008), 'Context change and travel mode choice: Combining the habit discontinuity and self-activation hypotheses', Journal of Environmental Psychology, 28 (2), pp. 121-127.

Warde, A. (2005), 'Consumption and theories of practice', Journal of Consumer Culture, 5(2): 131-54. Warde, A. (ed.) (2010), Consumption, Vols I-III, Sage Benchmarks in Culture and Society Series, Sage: London.

Warde, A., Cheng S-L., Olsen, W. and Southerton D. (2007), 'Changes in the practice of eating: a comparative analysis of time-use', Acta Sociologica, 50(4): 365-87.

Warde, A. \& Southerton, D. (2012), 'Social Sciences and Sustainability', in A. Warde \& D, Southerton (eds) 'The Habits of Consumption', Helsinki: Open Access Book Series of the Helsinki Collegium of Advanced Studies.

Whitford, J. (2002), 'Pragmatism and the untenable dualism of means and ends: why rational choice theory does not deserve paradigmatic privilege', Theory \& Society, 31 325-63.

Zerubavel, E. (1981), Hidden Rhythms: Schedules and Calendars in Social Life, Chicago: Chicago University Press. 


\section{Acknowledgements}

This article draws from research funded by the Sustainable Consumption Institute, The University of Manchester, and the ESRC co-funded 'Sustainable Practices Research Group' (RES-597-25-0003). I would particularly like to thank the members of the European Social Association's 'Consumption Research Network' for comments on a presentation of the embryonic form of this article, and Alan Warde, Elizabeth Shove and Andrew McMeekin for their various comments as that presentation was developed into this article.

Dale Southerton is Professor of Sociology and Research Director of the Sustainable Consumption Institute at the University of Manchester, UK. He is also Director of the ESRC co-funded Sustainable Practices Research Group. His research focuses on accounts of social change, particularly with reference to consumption. Recent research has addressed consumption in relation to sustainability, time use and temporality, theories of practice, innovation, and everyday life. 